\title{
Reviewer acknowledgement Journal of Polymer Engineering volume 40 (2020)
}

https://doi.org/10.1515/polyeng-2020-0311

The editors and the publisher wish to thank the following colleagues for their kind assistance in acting as referees for the journal in 2020: ${ }^{1}$

\begin{tabular}{|c|c|c|}
\hline Abdel Ghaffar, A. & Boddu, Sai & El-Gendi, Ayman \\
\hline Abdelmonem, Yasser & Borić, Andrej & Elmaaty, T. A. \\
\hline Abdul Majid, M. S. & Bressy, Christine & Erturun, Elif Kormali \\
\hline Aghjeh, Maosud Razavi & Bucio, Emilio & \\
\hline Agrawal, Alok & & Fakhrullin, Rawil \\
\hline Ahmad, Ayaz & Cacciotti, Ilaria & Fang, Huagao \\
\hline Akhter, Sohail & Cai, Qing & Faure-Vincent, Jérôme \\
\hline Alhathal Alanezi, Adnan & Campos, Alex Fabiano & Fechine, Guilhermino \\
\hline Ali, Imran & Cao, Weiyu & Feng, J. \\
\hline Al-Kalali, Naef A. & Cardinaels, Ruth & Feng, Yefeng \\
\hline Al-Salem, S. M. & Carrot, Christian & Filipe, Susana \\
\hline Alshdefat, Ramadan & Castro, Edison & Findik, Fehim \\
\hline Altay, Lutfiye & Catalin, Pintilie & Font, Josep \\
\hline Altstaedt, Volker & Cha, Sung Woon & Ford, David \\
\hline Amico, Sandro & Chaishome, Jedsada & Formela, Krzysztof \\
\hline Ansari, Seema & Chen, Jing & Fuoco, Alessio \\
\hline Anwar, Saleem & Chen, Xunjun & \\
\hline Aoki, Daisuke & Chen, Yu & Gao, Chao \\
\hline Armentano, Ilaria & Cheng, L. P. & Ge, L. Q. \\
\hline Atabaev, Timur & Choi, Seung Tae & Ghorbani, Farnaz \\
\hline \multirow[t]{2}{*}{ Aydınoğlu, Demet } & Chruściel, Jerzy J. & Giordano, Stefano \\
\hline & Chung, Jae Woo & Gokuldoss, Prashanth Konda \\
\hline Balandin, Alexander A. & Chung, Neal Tai-Shung & Gong, Jin \\
\hline Bárány, Tamás & Conceição, David & Grace, Landon \\
\hline Barille, Regis & Cui, Xiaoping & Gruzdev, Matvey \\
\hline Basaran, Ihsan & & Guido, Stefano \\
\hline Baskan, M. Halim & Dang, Xuan Phuong & Gull, Nafisa \\
\hline Bayraktar, Emin & Deshmukh, Kalim & Guo, Baolin \\
\hline Bednarek, Melania & Dong, Xuehui & Guo, Ruilan \\
\hline Beheshty, Mohammad Hosain & Doustgani, Amir & Gupta, Manoj \\
\hline Benedetti, Assis Vicente & Drioli, Enrico & \\
\hline Benli, Ahmet & Duan, Yugang & H., Devendrappa \\
\hline Benyoucef, Abdelghani & Dulebova, Ludmila & Han, C. Y. \\
\hline Bian, Jun & Dumee, Ludovic & Hanash, F. E. \\
\hline
\end{tabular}

1 The list covers the time span October 5, 2019 to November 8, 2020. 
Haque, Nasirul

Hassan, Azman

Hayeemasae, Nabil

He, Hongwei

He, Wei-Dong

Heli, Bentelhoda

Hendi, Awatif A.

Hojjati, Mehdi

Hossieny, Nemat

Huang, Farong

Ianniruberto, Giovanni

Incarnato, Loredana

Iqbal, Munawar

Ishigami, Akira

Jahan, Nusrat

Jahandideh, A.

Jalali Dil, Ebrahim

Jarukumjorn, Kasama

Jaymand, Mehdi

Jeong, Hoon Eui

Jia, Zhongfan

Jianghao, Qiao

Jihui, Wang

Johns, Jobish

Jong, Wen-Ren

Joshi, Shailesh

Kanagalakshmi, S.

Kanaya, Toshiji

Kandadai, Appan Srinivas

Kasmi, Nejib

Katakam, Prakash

Kavoosi, Gholamreza

Kesavan, K.

Khan, Ijaz Ahmad

Khatibi, Akbar A.

Kim, Hojoong

Klymov, Alexey

Kommavarapu, Pavan

Konovalov, Sergey

Koseko, Hisaaki

Krumme, Andres

Krumpfer, Joseph W.

Kuang, Tairong

Kuhn, Christoph

Kumar, D.

Lambiase, Francesco

Lamnawar, Khalid
Lee, Kee Hoon

Lemos, Mauricio Ferrapontoff

Li, Gang

Li, Guoqiang

Li, Jingye

$\mathrm{Li}, \mathrm{Li}$

Li, Shujian

Lin, Wei-Chih

Liu, Hua-Min

Liu, Xinli

Lopez-Sanchez, Patricia

Low, Siew Chun

Lu, Chang

Lucyshyn, Thomas

Ma, Songqi

Ma, Yao

Majtan, Juraj

Manickam, Sivakumar

Maras, M. Murat

Marques, Paula A. A. P.

Mascia, Leno

Masek, Anna

Massoud, Ayman

Matsuba, Go

Matsumoto, Toshihiko

Mayo, Latif

McKeown, Neil

Mehmet, Bulut

Mensitieri, Giuseppe

Mishra, Ajay K.

Moini Jazani, Omid

Moon, Joshua D.

Mukras, S. M. S.

Nam, Sang Yong

Natarajan, Upendra

Nayak, Nimai C.

Nishitsuji, Shotaro

Noor, Awal

Noor, Nuruzzaman

Norouzi, Sepideh

Nour, Abdelfattah Y. M.

Oh, Hee Jeung

Olad, Ali

Omer, A. M.

Osman, Azlin Fazlina

Ou, Jian Zhen

Özgür, Cihan
Pasquino, Rossana

Patel, Dinesh K.

Patel, Nirmal G.

Patil, Archana

Paulino, Alexandre

Pechyen, Chiravoot

Pereira, Carlos

Petchwattana, Nawadon

Pionteck, Jürgen

Pogorielov, Maksym

Pontes, Antonio

Poyraz, Bayram

Prasad, A. V. R.

Prochon, Miroslawa

Pulvirenti, Andrea

Qian, L. W.

Qin, Y. Y.

$\mathrm{Qu}$, Jin-ping

Raajeshkrishna, C. R.

Radziejewska-Trefon, Dominika

Ragaert, Kim

Rahman, Md.

Rahman, Mohammed MIzanur

Rangabhashiyam, S.

Read, Daniel

Redmann, Alec

Rezaei, Babak

Rota, Renato

Rupei, Tang

Sadak, Omer

Sailaja, R. R. N.

Salit, Mohd Sapuan

Sarti, Giulio

Savchenko, Valerii

Scaffaro, Roberto

Schubert, Dominik

Sekine, Tomohito

Shabbir, Maryam

Shahid, Salman

Shao, Yong

Sheng, Zhao Min

Shi, Xin Yan

Shimpi, Navinchandra G.

Shinji, Ando

Shioya, Masatoshi

Shunmugam, Raja

Sikora, Janusz

Silverwood, Richard 
Simon, Leonardo

Singh, Inderjeet

Sorrentino, Luigi

Spina, Roberto

$\mathrm{Su}$, Yongchao

Sukhadia, Ashish

Sultan, Mohamed Thariq $\mathrm{H}$.

Sun, Jingyao

Tarik Arafat, Muhammad

Tarpani, Jose

Tas, Mahmut

Thumsorn, Supaphorn

Tian, Huafeng

Tirillo, Jacopo

Tominaga, Yoichi Tominaga

Tosello, Guido

Tseng, Shi-Chang

Turng, Lih-Sheng

Vahabi, Henri

Vlachopoulos, John
Walters, Keisha

Wang, D. Y.

Wang, Hui

Wang, Ping

Wang, Q.

Wang, Shuanjin

Wang, Yixiang

Weng, Fei

With, Gijsbertus de

$\mathrm{Wu}, \mathrm{C}$. S.

$\mathrm{Wu}$, Dezhen

$\mathrm{Wu}$, Jin-Ming

$\mathrm{Wu}$, Wei

Xie, Sheng

$\mathrm{Xu}, \mathrm{Fu}$

Yadav, Mithilesh

Yamauchi, Yusuke

Yan, Long

Yang, Hao

Ye, Dezhan
Yokozeki, Tomohiro

Yu, Jae-Woong

Yuan, C. Q.

Yuya, Egawa

Zhai, Zhanyu

Zhang, Huihui

Zhang, Liaoyun

Zhang, Mao

Zhang, Rong

Zhang, Xiaohui

Zhang, Xinxing

Zhang, Yanhua

Zhang, Yong

Zhao, Gai

Zhao, Peng

Zhen, Weijun

Zhou, D.

Zhu, Shifeng

Zhu, Wenli 